\title{
EPILEPSIAS GENERALIZADAS IDIOPÁTICAS DIAGNOSTICADAS INCORRETAMENTE COMO EPILEPSIAS PARCIAIS
}

\author{
Susana B. Mory', Carlos A.M. Guerreiro' ${ }^{2}$ Li M. Li ${ }^{3}$, Ricardo A. Teixeira', \\ Alberto L.C. Costa ${ }^{4}$, Tânia A.M. Cardoso ${ }^{4}$, Fernando Cendes ${ }^{3}$
}

\begin{abstract}
RESUMO - A epilepsia generalizada idiopática (EGI) frequentemente não é diagnosticada corretamente em adultos, com sérias consequências para os pacientes. 0 objetivo deste estudo foi avaliar os fatores mais frequentemente associados a dificuldades no diagnóstico diferencial entre epilepsias parciais e generalizadas em adultos. Avaliamos 41 pacientes com diagnostico de crises parciais complexas com elementos de anamnese e EEG indicando um possível diagnóstico diferencial. Foi possível a mudança do diagnóstico de epilepsia parcial para EGI em 25 pacientes: 22 (88\%) com EMJ; um com ausência juvenil, um com síndrome de ausências com mioclonias periorais e um com ausência com mioclonias palpebrais. Mioclonias, uma das características da EMJ e outras formas de EGI, geralmente não eram espontaneamente relatadas pelos pacientes. Abalos mioclônicos unilaterais eram confundidos com crises parciais motoras. Ausências breves e pouco frequentes e anormalidades focais no EEG contribuíram para o não reconhecimento de EGI. Todos os 25 pacientes apresentavam crises sem controle adequado antes da revisão diagnóstica. Após o diagnóstico correto e mudança para monoterapia com acido valpróico ou valproato de sódio, 19 (76\%) ficaram livre de crises e seis (24\%) dos 25 pacientes apresentaram melhora significativa. A associação de lamotrigina em três destes pacientes propiciou redução significativa da frequência de crises. Em conclusão, anamnese detalhada e questionamento direcionado para determinar a presença de mioclonias e crises tipo ausência e a sua interpretação no contexto clínico são fundamentais para o diagnóstico correto das EGI em adultos.
\end{abstract}

PALAVRAS-CHAVE: epilepsia, diagnostico, tratamento, EEG.

Idiopathic generalized epilepsies misdiagnosed as partial epilepsies

\begin{abstract}
Idiopathic generalized epilepsy (IGE) is often not recognized with serious consequences on the sufferers. We examined factors contributing to the missed diagnosis of IGE in 41 adults attending our epilepsy clinic with diagnosis of partial epilepsy who had semiology or EEG findings suggesting a possible differential diagnosis. After careful re-evaluation, the diagnosis of IGE was established in 25 patients: 22 (88\%) with JME, one with juvenile absence, one with perioral myoclonia with absences, one with eyelid myoclonia with typical absences. Myoclonic jerks, the hallmark of the JME and other IGE, were not usually reported by patients or misdiagnosed as focal motor seizures. Brief and infrequent absence seizures and focal EEG abnormalities were other factors contributing to not recognizing JME. All 25 patients did not achieve seizure control before re-evaluation of diagnosis. After appropriate diagnosis of IGE and change of AED to valproate or valproic acid, $19(76 \%)$ became seizure free and six (24\%) had a significant improvement on seizure control. Association with lamotrigine provided further improvement in three of these patients. An appropriate questioning to identify myoclonic and absence seizures and a proper interpretation in the context of whole clinical constellation are essential for a correct seizure classification and diagnosis of IGE in adults.
\end{abstract}

KEY WORDS: epilepsy, diagnosis, treatment, EEG.

As crises epilépticas são classificadas de acordo com os critérios propostos pela Liga Internacional de Epilepsia (ILAE) em dois grandes grupos: parciais (ou focais) e generalizadas; estas por sua vez, são sub- divididas de acordo com as características da semiologia ictal e caracterização eletrencefalográfica ${ }^{1}$. As síndromes epilépticas também são classificadas em dois grandes eixos. No primeiro são divididas em par-

Departamento de Neurologia da Faculdade de Ciências Médicas da Universidade Estadual de Campinas (UNICAMP), Campinas SP, Brasil; ${ }^{1}$ Neurologista Pós-graduando; ${ }^{2}$ Livre Docente; ${ }^{3}$ Professor Assistente Doutor; ${ }^{4}$ Neurologista Assistente. Suporte financeiro: FAPESP.

Recebido 31 Janeiro 2002, recebido na forma final 3 Maio 2002. Aceito 16 Maio 2002. 
ciais (ou focais) e generalizadas; e no segundo eixo em idiopática/criptogênica e sintomática ${ }^{2}$. As crises parciais apresentam achados semiológicos e eletrencefalográficos que envolvem uma região específica do córtex cerebral ${ }^{1}$. As crises parciais são divididas em simples e complexas: nas crises parciais simples (CPS) o indivíduo é capaz de perceber e interagir com o meio, enquanto que nas crises parciais complexas (CPC) ocorre comprometimento da consciência.

Nas crises generalizadas, as primeiras manifestações clínicas indicam envolvimento de ambos os hemisférios e o padrão eletrográfico durante a crise é bilateral desde o início ${ }^{1}$. Dentre os diversos tipos de crises que ocorrem nas epilepsias generalizadas, as crises de ausência e as mioclônicas são as mais relevantes no contexto do diagnóstico diferencial entre as síndromes epilépticas em adultos. Nas crises de ausência ocorre diminuição ou perda da consciência com início e término súbitos, sem confusão pósictal. Clinicamente os pacientes apresentam olhar fixo e interrupção da atividade motora; em alguns casos podem apresentar automatismos e fenômenos autonômicos. Estas manifestações estão associadas a complexos espícula-onda lenta generalizados no eletroencefalograma (EEG) ${ }^{3,4}$.

Em alguns pacientes, o diagnóstico diferencial entre crises parciais complexas e crises de ausências pode ser difícil, levando a um diagnóstico inadequado da síndrome epiléptica ${ }^{3,5-7}$.

O objetivo deste estudo foi avaliar os fatores mais frequentemente associados a dificuldades no diagnóstico diferencial entre crises tipo ausências e mioclonias e crises parciais e assim tentar indicar elementos diagnósticos na rotina que possam ser úteis para o neurologista geral e epileptologista em fase de treinamento.

\section{MÉTODO}

Durante o período de janeiro de 2000 a julho de 2001, selecionamos de forma consecutiva pacientes do Ambulatório de Epilepsia do Hospital das Clínicas da Universidade Estadual de Campinas (HC/UNICAMP) que receberam diagnóstico de epilepsia parcial mas que apresentavam achados clínicos ou eletrencefalográficos sugerindo epilepsia generalizada idiopática (EGI) usando entrevistas estruturadas. Consideramos para este estudo o diagnostico inicial dos centros de atendimento primário e secundário por clínicos e neurologistas gerais que referiram os pacientes para o Ambulatório de Epilepsia do HC/UNICAMP (incluindo esquema terapêutico prévio que o paciente utilizava antes de ingressar no nosso serviço). Avaliamos também pacientes do nosso ambulatório com diagnóstico inicial equivocado devido a razões diversas discutidas no decorrer do trabalho.
Os critérios para seleção inicial dos pacientes foram: pacientes com idade superior a 14 anos; crises classificadas como parciais complexas e/ou simples motora que apresentam EEG interictal com atividade de base normal e complexos espícula-onda lenta; pacientes com diagnóstico de crises parciais complexas que não respondem bem ao tratamento medicamentoso e que apresentam episódios suspeitos de mioclonias; exame neurológico normal.

Os critérios de exclusão estabelecidos foram: co-existência de outra doença neurológica além da epilepsia afetando o sistema nervoso central (SNC); gravidez; não consentimento para o estudo.

Realizamos a revisão e entrevista estruturada com os pacientes e responsáveis, com especial atenção para os seguintes aspectos: 1) Caracterização das crises epilépticas: início de crises, tipo e classificação, descrição detaIhada, frequência, horário de crises, fatores predisponentes, como stress, privação de sono, ingesta de bebida alcoólica, abstinência de droga, período menstrual; 2) Esquemas terapêuticos prévios e atuais bem como a eficácia de cada tratamento (critérios avaliados pelo próprio paciente e familiares); 3)Antecedentes pessoais: convulsão febril, convulsão neonatal, antecedentes natais e perinatais, traumatismo crânio-encefálico acompanhado de perda de consciência; 4) Antecedentes familiares: epilepsia (idade de início, tipo de crises), outras doenças neurológicas; 5) Exame físico e neurológico; 6) Investigação eletrencefalográfica; 7) Diagnóstico inicial pelo prontuário e diagnóstico final do paciente após revisão detalhada dos dados enumerados acima; 8) Evolução clínica do paciente após esta re-avaliação e mudança na prescrição de anticonvulsivantes.

EEG - A investigação eletrencefalográfica consistiu em EEGs interictais com a colocação de eletrodos segundo o sistema internacional 10-20 e manobras de ativação padronizadas em aparelho de EEG analógico de 16 canais usando diferentes montagens bipolares e referenciais ${ }^{8}$.

Ressonância magnética (RM) - As imagens de RM foram obtidas em sistema de RM de 2 Tesla com aquisições nos planos coronal, sagital e axial além de aquisição 3D (volumétrica) que permite reconstrução das imagens a posteriori em qualquer plano ou inclinação.

Os parâmetros de imagem foram: 1) Imagens sagitais T1 ponderadas "spin-echo" (espessura de $6 \mathrm{~mm}$, ângulo de excitação de $180^{\circ}, \mathrm{TR}=430, \mathrm{TE}=12$ matriz de $200 \times 350$, $\mathrm{FOV}=25 \times 25 \mathrm{~cm}$ ); 2) Imagens no plano coronal oblíquo perpendiculares ao longo do eixo da formação hipocampal, definido nas imagens sagitais; 2 a) T2 ponderadas "fast spin echo" (espessura de $4 \mathrm{~mm}$, ângulo de excitação de $120^{\circ}$, $\mathrm{TR}=5800 ; \mathrm{TE}=129$; matriz de $252 \times 320 ; \mathrm{FOV}=18 \times 18 \mathrm{~cm}$ ); 2.b) T1 ponderadas inversion recovery (espessura de $3 \mathrm{~mm}$, ângulo de excitação de $200^{\circ}$; TR IR=2800; TE= $14 \mathrm{TI}=840$ matriz $130 \times 256$; FOV $=16 \times 18 \mathrm{~cm}$ ); 3) Imagens no plano axial: duplo spin echo (T2 ponderadas e densidade de prótons): T2 ponderadas (espessura de $6 \mathrm{~mm}$, ângulo de excitação $180^{\circ} \mathrm{TR}=1800, \mathrm{TE}=90$, matriz $165 \times 256 ; \mathrm{FOV}=20 \times 24$ 
$\mathrm{cm}$ ); densidade de prótons (espessura de $6 \mathrm{~mm}$, ângulo de excitação de $180^{\circ}, \mathrm{TR}=1800, \mathrm{TE}=16$, matriz 165 x 256; $\mathrm{FOV}=20 \times 24 \mathrm{~cm}$ ou "fast spin echo" $\mathrm{T} 2$ ponderadas (espessura de $4 \mathrm{~mm}$; ângulo de excitação de $120^{\circ}$, TR $=6800$; $\mathrm{TE}=129$; matriz de $252 \times 328 ; \mathrm{FOV}=21 \times 23 \mathrm{~cm}$ ) e 4) Aquisição 3D obtida no plano sagital "gradiente echo" T1 ponderadas (espessura $1 \mathrm{~mm}$, ângulo de excitação de 350; $\mathrm{TR}=22$; $\mathrm{TE}=9$, matriz $256 \times 220 \mathrm{FOV}=23 \times 25 \mathrm{~cm}$; pixel= 1×1).

O protocolo estudado foi aprovado pelo Comitê de Ética Médica da Faculdade de Ciências Médicas da UNICAMP. Todos pacientes assinaram termo de consentimento para o estudo.

\section{RESULTADOS}

Selecionamos um grupo de 41 pacientes com crises inicialmente diagnosticadas como parciais complexas mas com elementos para um possível diagnóstico diferencial com ausências e/ou mioclonias seguindo os critérios de inclusão e exclusão acima.

Utilizando os critérios da ILAE foi possível a reclassificação do diagnóstico sindrômico de epilepsia parcial para EGI em 25 pacientes. Destes, 22 (88\%) receberam o diagnóstico de epilepsia mioclônica juvenil (EMJ). As características clínicas destes pacientes estão resumidas na Tabela 1. O Paciente 6 foi classificado como provável síndrome de ausência com mioclonias periorais ${ }^{9}$, a Paciente 5 como provável síndrome de ausência com mioclonias palpebrais ${ }^{10}$ e o Paciente 9 como ausência juvenil.

Em 16 pacientes o diagnóstico de epilepsia parcial foi confirmado. O resultado da investigação eletrencefalográfica e RM destes pacientes estão resumidos na Tabela 2.

\section{Dados demográficos e antecedentes}

A média de idade do início das crises destes 25 pacientes (13 mulheres) foi de 12,6 anos, variando de 5 a 22 anos. Treze (52\%) pacientes (todos com diagnóstico de EMJ) apresentavam antecedente de epilepsia em familiares de primeiro grau. Apenas um paciente com EMJ apresentou antecedente de convulsão febril na infância (Paciente 2 ).

As crises de ausência foram descritas pelos pacientes e acompanhantes como: 1) "sensação de desligamento", 2) "gagueira", 3) "olhar parado", 4) "sai do ar" 5) "apagar de uma luz"; e as crises mioclônicas como: 6) tremores em membros uni ou bilateralmente, 7) "repuxamento" do braço, 8) "choques", 9) "sensação de calafrios", 10) "queda de objetos" (Tabela 1).

Em nove dos 25 pacientes (36\%) as mioclonias inicialmente foram identificadas como crises parciais simples motoras. Dois destes 9 pacientes (Pacientes 10 e 20) tiveram diagnóstico clínico inicial de epilepsia benigna da infância por apresentarem mioclonias periorais na infância.

\section{Eletroencefalograma}

Observamos distúrbio epileptiforme focal associado a distúrbio epileptiforme generalizado em 8 pacientes (32\%). O diagnóstico inicial destes 8 pacientes foi de epilepsia parcial. Destes, 7 foram reclassificados como EMJ e uma (Paciente 5) como epilepsia com mioclonias palpebrais.

\section{Resposta terapêutica}

Todos os 25 pacientes apresentavam crises sem controle adequado antes da revisão diagnóstica. Após correção do diagnóstico e mudança do regime terapêutico para monoterapia com valproato de sódio (ou acido valpróico), 19 (76\%) ficaram livre de crises. No entanto, 5 destes 19 pacientes livres de crise com ácido valpróico ou valproato de sódio necessitaram de mudança de anticonvulsivante devido a efeitos colaterais ou dificuldades econômicas; no momento estão em uso de oxcarbazepina (Paciente 16), carbamazepina (Pacientes 7 e 12) e fenitoína ( Pacientes 3 e 18).

Seis $(24 \%)$ dos 25 pacientes apresentaram melhora significativa após novo regime terapêutico, porém continuaram apresentando mioclonias e crises de ausência apesar de doses adequadas e boa aderência ao ácido valpróico em monoterapia.

Foi realizada então a associação de lamotrigina em três pacientes (Pacientes 11, 23 e 25), com redução significativa da frequência de crises na Paciente 23 e persistência das mioclonias esporádicas nos Pacientes 11 e 25.

O Paciente 6 está em uso de ácido valpróico associado a carbamazepina e clonazepam e apresenta também mioclonias esporádicas.

A Paciente 15 persiste com mioclonias e ausências esporádicas mesmo com doses adequadas de ácido valpróico. Nesta paciente, o uso de lamotrigina foi descontinuado, devido a reação alérgica.

A Paciente 5 descontinuou o tratamento com valproato de sódio e preferiu retornar ao uso de carbamazepina; continua com crises ausências esporádicas e persistência de EEGs com distúrbio epileptiforme generalizado.

\section{Ressonância magnética}

Onze destes 25 pacientes foram submetidos a RM de alta resolução e análise detalhada, incluindo reconstrução multiplanar; os exames foram considerados normais em todos eles.

Fatores que influenciaram no diagnóstico entre crises parciais e generalizadas

O tempo para o diagnóstico diferencial entre epi- 
Tabela 1 . Características cliínicas.

\begin{tabular}{|c|c|c|c|c|c|c|}
\hline Pac & Sexo & Idade & Início & Descrição das crises* & $\begin{array}{l}\text { Fator } \\
\text { desencadeante }\end{array}$ & $\begin{array}{l}\text { História } \\
\text { familiar }\end{array}$ \\
\hline 1 & M & 37 & 22 & desligamentos, tremor & & não \\
\hline 2 & M & 35 & 16 & olhar fixo c/automatismos & & $\operatorname{sim}$ \\
\hline 3 & M & 29 & 14 & tremor dos braços, piscamentos bilaterais & & não \\
\hline 4 & M & 30 & 14 & desligamentos, tremor & & não \\
\hline 5 & $\mathrm{~F}$ & 20 & 5 & desligamento desencadeado pela luz & luz solar & não \\
\hline 6 & M & 48 & 15 & olhos abertos, foge os pensamentos, repuxa rima $E$ & & não \\
\hline 7 & $\mathrm{~F}$ & 18 & 11 & choque, desligamento, repuxamento de MSD & período menstrual & $\operatorname{sim}$ \\
\hline 8 & $\mathrm{~F}$ & 36 & 14 & dificuldade de fala (gagueira) c/ contração de MSD & & não \\
\hline 9 & M & 20 & 13 & olhar parado & & não \\
\hline 10 & M & 15 & 8 & gagueira+ desvio de rima, abalos & & $\operatorname{sim}$ \\
\hline 11 & M & 29 & 12 & tremores antes das crises, sensação de susto, sensação ruim & & não \\
\hline 12 & $\mathrm{~F}$ & 27 & 10 & repuxamento de MSD & privação de sono & $\operatorname{sim}$ \\
\hline 13 & M & 36 & 15 & repuxamento de MSD, gagueira & & $\operatorname{sim}$ \\
\hline 14 & $\mathrm{~F}$ & 44 & 7 & desligamento súbito, abalos nos membros & & não \\
\hline 15 & $\mathrm{~F}$ & 28 & 14 & sensação de vazio, olhar fixo & privação de sono & $\operatorname{sim}$ \\
\hline 16 & $\mathrm{~F}$ & 17 & 14 & desligamento de curta duração, repuxamento & & $\operatorname{sim}$ \\
\hline 17 & $\mathrm{~F}$ & 24 & 11 & foge os pensamentos, CTCG durante o sono, tremor em MMSS & & $\operatorname{sim}$ \\
\hline 18 & M & 36 & 13 & gagueira+ repuxamento de MMSS & & não \\
\hline 19 & M & 31 & 17 & repuxamento de MMSS, alheio ao meio ambiente e tontura & & $\operatorname{sim}$ \\
\hline 20 & M & 20 & 11 & repuxamento de rima $D$ & & não \\
\hline 21 & $\mathrm{~F}$ & 25 & 11 & desligamento, derruba objetos & & $\operatorname{sim}$ \\
\hline 22 & $\mathrm{~F}$ & 30 & 21 & desligamento, repuxamento de MSE & privação de sono & $\operatorname{sim}$ \\
\hline 23 & $\mathrm{~F}$ & 33 & 6 & desligamento, tremor de extremidades & & $\operatorname{sim}$ \\
\hline 24 & $\mathrm{~F}$ & 36 & 13 & desligamento, queda de objetos, & & não \\
\hline 25 & M & 47 & 7 & desligamento, queda do pescoço, tremor em mãos & & $\operatorname{sim}$ \\
\hline média & & 30,0 & 12,6 & & & \\
\hline DP & & 9,0 & 4,2 & & & \\
\hline
\end{tabular}

* Todos os pacientes apresentavam CTCG; MMSS, membros superiores; MSD, membro superior direito; MSE, menbro superior esquerdo.

lepsia parcial e EGI (incluindo o subtipo EMJ) foi 4,6 $( \pm 3,1)$ anos.

Os fatores que colaboraram para o diagnóstico preciso estão resumidos na Tabela 3 e aqueles que colaboraram para um diagnóstico equivocado estão resumidos na Tabela 4.

A Tabela 5 inclui os resultados de EEGs, medicações antes e depois do diagnóstico de EGI.

\section{DISCUSSÃO}

As CPCs originárias do lobo frontal podem ser parecidas com as crises de ausências quando apresentam curta duração, alta frequência durante o dia e ausência de confusão pós-ictal ${ }^{7,11-13}$.

As crises parciais representam o tipo de crise mais frequente em adultos, no entanto a frequência da crises de ausência em adultos é geralmente subestimada, o 
Tabela 2. EEG, terapêutica, Vídeo-EEG e imagem do Grupo 2.

\begin{tabular}{|c|c|c|c|c|}
\hline Pac & EEGs interictais & Medicação atual & Vídeo-EEG & RM \\
\hline 26 & CE e PEOLIG/ & $\mathrm{LMT}+\mathrm{VPA}$ & $\begin{array}{l}\text { Poliespículas } 1-4 \mathrm{~Hz} \\
\text { seguidas de PEOLG }\end{array}$ & displasia frontal Dir. \\
\hline 27 & CEOLR $3 \mathrm{HZ}$ & $\mathrm{CBZ}$ & & polimicrogiria perisilviana a Dir \\
\hline 28 & CEOLG /EF Fr-Temp E & $\mathrm{CBZ}+\mathrm{VPA}+\mathrm{CLZ}$ & CEOLIG & displasia frontal bilateral \\
\hline 29 & CEOLG máx FTD,/OA Fr-Temp. D & $\mathrm{CBZ}$ & & displasia frontal Dir. \\
\hline 30 & EF Reg. Par. Dir. e C PEOLIG & oxc & & displasia frontal Dir. \\
\hline 31 & $\begin{array}{l}\text { OF e CE e PEOL Reg.Temp. Dir / } \\
\text { e CE e PEOL G }\end{array}$ & CBZ1200 & & $\begin{array}{l}\text { polimicrogiria perisilviana } \\
\text { direita }\end{array}$ \\
\hline 32 & EF Reg. Temp. Esq e CEOLIG & CBZ & & $\begin{array}{l}\text { cisto aracnóide polo temp. } \\
\text { Esq }+ \text { malformação lobo } \\
\text { temp.e frontal esq }\end{array}$ \\
\hline 33 & EF Fr. Dir e CEOLIG / CEOLIG & VPA 1500 & & $\begin{array}{l}\text { lesão no polo occipital } \\
\text { dir (gliose) }\end{array}$ \\
\hline 34 & EFFr. Dir. e EF Fr. Esq e CEOLIG/COAOLIG & $\mathrm{CBZ}+\mathrm{CLB}$ & & heterotopia laminar em banda \\
\hline 35 & CEOLI 3-4 Hz/ EF Fr. Dir D e CEOLIG & VPA + LMT & $\begin{array}{l}\text { Mioclonias sem } \\
\text { alterações no EEG }\end{array}$ & displasia frontal dir. \\
\hline 36 & EF Fr. E e CEOLIG/EF Fr. Dir. e CEOLIG & $\mathrm{VPA}+\mathrm{CBZ}$ & $\begin{array}{l}\text { Políespiculas G } \\
\text { seguidadas de CEOL I G }\end{array}$ & displasia frontal dir. \\
\hline 37 & EF Fr. Dir e EF Fr. Esq e CEOLIG/COAOLIG & $\mathrm{VPA}+\mathrm{CBZ}+\mathrm{CLB}$ & $\begin{array}{l}\text { OA F reg. Temp. Esq. } \\
\text { e AO F reg. Temp. Dir } \\
\text { precedendo COAOL G }\end{array}$ & displasia frontal bilateral \\
\hline 38 & $\begin{array}{l}\text { COAL /OAF Fr.- Temp E e } \\
\text { CEOLIG/ CE e PEOL G }\end{array}$ & $\mathrm{CBZ}+\mathrm{CLB}$ & $\begin{array}{l}\text { CE e PEOL I G 2-4hz reg. } \\
\text { Fr.Cent.Temp Esq }\end{array}$ & atrofia hipocampal E+LTE \\
\hline 39 & EF Fr. Dir e EF Fr. Esq e CEOLIG/COAOL I G & $\mathrm{CBZ}+\mathrm{CLB}$ & & displasia frontal esq. \\
\hline 40 & CEOL I G & $\mathrm{FNT}, \mathrm{VPA}, \mathrm{CBZ}+\mathrm{CLB}$ & & displasia frontal dir. \\
\hline 41 & OAF Reg. Temp. Dir e Esq. e CEOILG & VPA+LMT & $\begin{array}{l}\text { Atividade teta rírmica } \\
\text { em regiões frontais }\end{array}$ & heterotopia laminar em banda \\
\hline
\end{tabular}

CEOL, complexos espícula-onda lenta; I, irregulares; G, generalizados; PEOL, poliespícula onda-lenta; COAOL, complexo ondas aguda - onda lenta; EF, espícula focal; OAF, onda aguda focal; Fr, frontal; Temp, temporal; Par, parietal.

que colabora para o erro de interpretação do médico quando o paciente ou acompanhante fornecem uma descrição semiológica inadequada das crises ${ }^{3,14}$.

Outro dado que pode favorecer o erro no diagnóstico são as anormalidades focais epileptiformes em pacientes com epilepsia generalizada primária 5 . Lombroso ${ }^{15}$ estudou os padrões eletrencefalográficos em 58 pacientes com epilepsia generalizada primária durante duas décadas e observou que em 32 (56\%) desses 58 pacientes, 65\% dos EEGs apresentavam anormalidades focais, incluindo ondas lentas na frequência delta e teta, ondas agudas e espículas. Neste estudo, as anormalidades eletrencefalográficas focais foram encontradas principalmente em regiões temporais e menos frequentemente em regiões frontais. Porém, segundo Lombroso, as anormalidades epileptiformes focais nestes pacientes são pouco definidas e variáveis na localização ${ }^{15}$.

Panayiotoupolos e col. ${ }^{3}$ relataram que as mioclonias podem ser confundidas com crises parciais simples motoras na EMJ. Além disso, observaram que um terço de pacientes adultos com crises de ausência típica e EMJ foram classificados previamente como CPCs por clínicos e neurologistas experientes. Esses autores descreveram anormalidades focais nos EEGs e ressaltaram que estas poderiam ser interpretadas como indicativas de CPCs. Outros estudos também que demonstraram anormalidades focais na EMJ ${ }^{5,16,17}$ bem como diagnósticos equivocados ${ }^{18}$. 
Tabela 3. Fatores que colaboraram para o diagnóstico adequado.

\begin{tabular}{|c|c|c|c|}
\hline a) & EEGs seriados & 2 pacientes & $8 \%$ \\
\hline b) & história de mioclonias a partir de busca ativa e história familiar & 1 paciente & $4 \%$ \\
\hline c) & EEG com história de mioclonias (bilaterais) a partir de busca ativa & 8 pacientes & $32 \%$ \\
\hline d) & EEG com história de mioclonias unilaterais (antes classificadas como crises parciais simples motoras) & 4 pacientes & $16 \%$ \\
\hline e) & EEG com antecedente familiar e história de mioclonias (bilaterais) a partir de busca ativa & 5 pacientes & $20 \%$ \\
\hline f) & $\begin{array}{l}\text { EEG com antecedente familiar positivo e história de mioclonias unilaterais (antes classificadas como } \\
\text { crises parciais simples motoras) }\end{array}$ & 5 pacientes & $20 \%$ \\
\hline
\end{tabular}

Tabela 4. Fatores que colaboraram para um diagnóstico equivocado.

\begin{tabular}{|c|c|c|c|}
\hline a) & Anamnese (CPC) & 3 pacientes & $12 \%$ \\
\hline b) & EEG normais persistindo a impressão diagnóstica inicial deCPC & 10 pacientes & $40 \%$ \\
\hline c) & EEG com anormalidade epileptiforme focal (pelo menos um) e anamnese levando & & \\
\hline & ao diagnóstico de CPC & 3 pacientes & $12 \%$ \\
\hline d) & EEG normal associado a história de crise parcial simples motora e complexa(CPSM/CPC) & 3 pacientes & $12 \%$ \\
\hline e) & $\begin{array}{l}\text { EEG com anormalidade epileptiforme focal e anamnese (mioclonias assimétricas e unilaterais } \\
\text { interpretadas como crises parciais simples motoras) }\end{array}$ & 5 pacientes & $20 \%$ \\
\hline f) & EEG com anormalidades inespecificas e historia de CPC & 1 paciente & $4 \%$ \\
\hline
\end{tabular}

Na sua maioria, nossos pacientes (88\%) foram re-classificados em EMJ. A EMJ é um tipo relativamente frequente de epilepsia, representando 7 a $9 \%$ das epilepsias ${ }^{17,19}$ com início entre os 8 e 26 anos de idade e em $85 \%$ dos casos entre os 13 e 20 anos $^{17,19,20}$. O melhor conhecimento da síndrome tem contribuído para o aumento da taxa de prevalência referida na literatura, pois tanto pacientes como médicos frequentemente subestimam o significado das mioclonias $^{17,19,20}$. A EMJ acomete igualmente homens e mulheres e apresenta história familiar positiva em $17-50 \%$ dos $\operatorname{casos}^{16,19}$. A síndrome caracteriza-se por mioclonias durante o despertar, CTCG e ausências em $14,6 \%$ a $39,5 \%{ }^{16,21}$. O padrão eletrencefalográfico interictal consiste em complexos-espícula onda-lenta irregulares generalizados, com frequência entre 3,5$4,5 \mathrm{~Hz}$, ocasionalmente limitados às regiões frontais ${ }^{16}$.

Nossos pacientes relatavam frequentemente "tremores em membros superiores", "sensação de calafrios", "choques", "repuxamento de um membro", "queda de objetos". Tais sintomas são compatíveis com as mioclonias da EMJ que podem ser simétricas ou assimétricas, únicas ou repetitivas, rápidas e de amplitude variável, sempre mais pronunciados e frequentemente restritos aos membros superiores. Estas mioclonias podem acometer os membros inferiores ocasionando queda, ou ainda a musculatura cervical e, excepcionalmente, a face como aconteceu com os nossos Pacientes 10 e $20^{17}$.

Segundo o estudo de Panayiotoupolos e col. ${ }^{4}$, as crises de ausência típica podem ser descritas pelo paciente como "blackouts", "obnubilação" ou "fuga de pensamento". De acordo com o subtipo de epilepsia (ausência infantil, juvenil e ausência da EMJ) há um padrão eletrográfico típico. As crises de ausência da EMJ geralmente são de curta duração e pouco frequentes, ao contrário das ausências infantil e juvenil, que são mais prolongadas ${ }^{4}$.

Somente um paciente (Paciente 2 ) (4\%) do grupo com o diagnóstico de EMJ apresentou convulsão febril na infância. Segundo Janz ${ }^{16}$, convulsões febris podem ser mencionadas na história de pacientes com EMJ e ocorrem em 4,4\%, dado equivalente à frequência da população geral.

Os Pacientes 10 e 20 apresentaram diagnostico inicial de epilepsia benigna da infância provavelmente devido às mioclonias periorais assimétricas. O Paciente 10 apresentou EEG evidenciando anormalidades focais em região centro-temporal esquerda além de anormalidades generalizadas, com posterior evolução durante a adolescência para mioclonias segmentares em membro superior direito. Já no Paciente 20, o EEG evidenciou anormalidade focal em 
Tabela 5. EEG, drogas, intervalo para diagnóstico e diagnóstico final.

\begin{tabular}{|c|c|c|c|c|c|}
\hline Pac & EEGs & DAE anteriores & DAE atual & $\begin{array}{l}\text { Intervalo } \\
\text { diagnóstico }\end{array}$ & $\begin{array}{l}\text { Diagnóstico } \\
\text { final }\end{array}$ \\
\hline 1 & CEOL I G & CBZ & VPA & 5,0 & EMJ \\
\hline 2 & $\begin{array}{l}\text { EF Fr. Esq; OAF Temp. Dir e Esq; } \\
\text { CEOL I G }\end{array}$ & $\mathrm{CBZ}$, FNT, CBZ+FNT, VPA+CBZ & VPA & 6,0 & EMJ \\
\hline 3 & CEOL I G & $\mathrm{CBZ}+\mathrm{FNT}+\mathrm{PB}, \mathrm{PB}, \mathrm{CBZ}, \mathrm{VPA}$ & FNT & 7,0 & EMJ \\
\hline 4 & CEOL I G & $\mathrm{CBZ}, \mathrm{PB}$ & VPA & 2,0 & EMJ \\
\hline 5 & $\begin{array}{l}\text { CEOL I G; OAF Reg Parietal Dir } \\
\text { e Esq; OAF Reg Fr-Temp Esq }\end{array}$ & CBZ, Primidona, VPA & $\mathrm{CBZ}$ & 8,0 & EGP \\
\hline 6 & CEOL e PEOL G & $\mathrm{VPA}+\mathrm{CBZ}+\mathrm{CLZ}$, FNT, Comital L, PB & $\mathrm{VPA}+\mathrm{CBZ}+$ rivotril & 7,0 & EGP \\
\hline 7 & CEOL G $3 \mathrm{hz}$ & CBZ, VPA & $\mathrm{CBZ}$ & 2,0 & EMJ \\
\hline 8 & CEOL I G; AOF Temp Dir & $\mathrm{PB}, \mathrm{FNT}, \mathrm{CBZ}+\mathrm{VPA}$ & VPA & 12,0 & EMJ \\
\hline 9 & CEOL I G & $C B Z+V P A$ & VPA & 0,6 & EGP \\
\hline 10 & $\begin{array}{l}\text { CEOL e PEOL Fr-Ct. Esq; CEOL } \\
\text { e PEOL I G }\end{array}$ & FNT & VPA & 5,0 & EMJ \\
\hline 11 & CE e PEOL Fr-Temp Esq; CEOL I G & $\mathrm{PB}, \mathrm{FNT}, \mathrm{CBZ}, \mathrm{VPA}+\mathrm{FNT}$ & VPA+LMT & 6,0 & EMJ \\
\hline 12 & CEOL I G; OAF Fr-Temp Dir & $\mathrm{PB}, \mathrm{FNT}, \mathrm{CBZ}, \mathrm{VPA}$ & $\mathrm{CBZ}$ & 1,0 & EMJ \\
\hline 13 & CEOL e PEOL I G & $\mathrm{PB}, \mathrm{FNT}, \mathrm{CBZ}$ & VPA & 4,0 & EMJ \\
\hline 14 & OLI G & FNT,PB,Nitrazepan, VPA+CBZ+ Rivotril & VPA & 2,0 & EMJ \\
\hline 15 & CEOL G $3 \mathrm{hz}$ & $\mathrm{PB}, \mathrm{CBZ}$ & VPA & 9,0 & EMJ \\
\hline 16 & CEOL I G & OCBZ, VPA & Oxcarbazepina & 2,0 & EMJ \\
\hline 17 & CE e PEOL I G & $\mathrm{PB}+\mathrm{FNT}+\mathrm{CBZ}, \mathrm{CBZ}$ & VPA & 1,0 & EMJ \\
\hline 18 & CE e PEOL I G & CBZ,VPA & FNT & 8,0 & EMJ \\
\hline 19 & CEOL I G e OAF Fr-Temp Esq & PB, FNT,VPA & VPA & 5,0 & EMJ \\
\hline 20 & CE e PEOL I G; EF Fr. Esq & FNT, VPA, PB.CBZ & VPA & 8,0 & EMJ \\
\hline 21 & CEOL I G; EF Fr. Esq & $\mathrm{PB}, \mathrm{FNT}, \mathrm{CBZ}, \mathrm{VPA}+\mathrm{CLB}$ & VPA & 1,0 & EMJ \\
\hline 22 & CEOL I G & $\mathrm{CBZ}+\mathrm{VPA}$ & VPA & 4,0 & EMJ \\
\hline 23 & CEOL G 4Hz; OLI Temp Esq e Dir & $\mathrm{CBZ}+$ Comital+VPA, $\mathrm{CBZ}$ & $\mathrm{LMT}+\mathrm{VPA}$ & 2,0 & EMJ \\
\hline 24 & CEOL I G & $\mathrm{CBZ}, \mathrm{CBZ}+\mathrm{VPA}$ & VPA & 6,0 & EMJ \\
\hline \multirow[t]{3}{*}{25} & CEOL I G & PB, FNT, CBZ, VPA & $\mathrm{LMT}+\mathrm{VPA}$ & 1,0 & \\
\hline & & & Média & 4,6 & \\
\hline & & & DP & 3,1 & \\
\hline
\end{tabular}

CEOL, complexos espícula onda lenta; I, irregulares; G, generalizados; PEOL, poliespícula onda lenta; OLI, ondas lentas intermitentes; OAF, onda aguda focal; EF, espículas focais; Fr, frontal; Temp, temporal; Ct., central.

região fronto-temporal esquerda além de complexos espícula e poli-espícula onda lenta generalizados. Estes dois pacientes estão controlados com ácido valpróico em monoterapia.

Os nossos Pacientes 10 e 20 apresentavam vários EEGs evidenciando complexos tipo poli-espícula onda lenta irregulares generalizados e relato de mioclonias segmentares na adolescência, com bom controle das crises. O quadro inicial de ambos não é compatível com o dos pacientes descritos por Capovilla e col. ${ }^{22}$ devido à alteração epileptiforme generalizada irregular, nem com a síndrome das au- 
sências com mioclonias periorais ${ }^{9}$ devido à boa resposta terapêutica e às mioclonias segmentares em membros; portanto, foram classificados como EMJ.

As mioclonias periorais podem ocorrer em crianças com epilepsia ausência infantil e EEG com complexos tipo espícula onda lenta regulares generalizados na frequência de $3 \mathrm{~Hz}^{22}$. Por outro lado, a síndrome descrita por Panayiotoupolos ${ }^{9}$, denominada ausências com mioclonias periorais é mais frequente em adultos e apresenta complexos tipo espícula e poli-espícula onda lenta irregulares generalizados, além de difícil controle das mioclonias e história familiar positiva. No Paciente 6 , devido a persistência de EEGs com complexos tipo poli-espícula-onda lenta irregulares generalizados, presença de mioclonias de rima de difícil controle e ressonância magnética normal, o diagnóstico foi síndrome de ausências com mioclonias periorais.

Na Paciente 5, a história de ausência desde os 6 anos de idade acompanhada de piscamentos e desencadeada pela luz do sol, EEGs com complexos tipo espícula e poli-espícula onda lenta irregurales generalizados com resposta fotoparoxística, refratariedade ao ácido valpróico e valproato de sódio, levaram ao diagnóstico de síndrome de ausência com mioclonias peripalpebrais ${ }^{10}$.

O tempo para o diagnóstico diferencial entre epilepsia parcial e EGI (incluindo o subtipo EMJ) foi 4,6 $( \pm 3,1)$ anos. Este dado está de acordo com Panayiotopoulos e col. ${ }^{3}$ que encontraram intervalo de 8,3 ( \pm 5.5) anos entre diagnóstico inicial e diagnóstico adequadro de EMJ.

Observamos que as mioclonias raramente eram mencionadas pelos pacientes de forma espontânea e somente foi feito o diagnóstico a partir de interrogatório sistemático da semiologia ictal e busca objetiva de anormalidades em EEGs seriados e história familiar. Outro fato importante é que os pacientes com EMJ (e também os médicos) frequentemente dão maior importância às mioclonias unilaterais, 0 que colabora com o diagnóstico equivocado de crises parciais simples motoras.

No caso das ausências, os pacientes descreviam desligamentos e em nenhum deles houve o relato de automatismos, confusão ou amnésia pós-ictal.

Observamos também que a impressão diagnóstica inicial, direcionada ao fato de que as crises parciais são as mais frequentes em adultos, associada ou não a um EEG focal, contribuiu para o retardo no diagnóstico em $88 \%$ dos pacientes. Outro fator que pode dificultar o diagnóstico diferencial é o fato de que os EEGs de rotina em vigília frequentemente não apresentam anormalidades definidas em adultos com EGI. EEGs de rotina nas primeiras 24 horas após uma crise ou após privação de sono mostram anormalidades epileptiformes mais frequentemente ${ }^{23}$.

As causas de ocorrência de padrão eletrográfico focal em epilepsias generalizadas até o momento não estão bem esclarecidas. Uma hipótese muito discutida é o conceito de "microdisgenesia" cortical descrito por Meencke e col. ${ }^{24}$. Porém, este conceito é controvertido ${ }^{25}$.

Em relação à resposta terapêutica, embora alguns pacientes do nosso grupo tenha apresentado bom controle de crises com o uso da carbamazepina e fenitoína, tal fato não é a regra. Genton e col. ${ }^{26}$ observaram que $15 \%$ dos pacientes estudados com EMJ apresentaram melhora das crises com o uso da carbamazepina em monoterapia.

A carbamazepina (provavelmente também a oxcarbazepina) e a fenitoína podem agravar tanto as crises de ausência quanto as mioclonias ${ }^{14,26-29}$. Portanto, o uso destas medicações em pacientes com EGl em geral não controla adequadamente as crises podendo paradoxalmente aumentar a frequência de crises, levando a aumento progressivo das dosagens ou uso de politerapia, proporcionando aumento dos efeitos colaterais e qualidade de vida insatisfatória .

O tratamento de escolha das EGI, em particular da EMJ, é o ácido valpróico ou valproato de sódio, que possibilita o controle completo de todos os tipos de crises nesta síndrome em $90-95 \%$ dos pacientes ${ }^{16}$.

Em conclusão, um alto índice de suspeita, a anamnese detalhada e o questionamento direcionado para determinar a presença de mioclonias e crises tipo ausência e história familiar são fundamentais para o diagnóstico correto das EGI em adultos, antes mesmo de avaliar os exames complementares como o EEG.

\section{REFERÊNCIAS}

1. Commission on Classification and Terminology of the International League Against Epilepsy. Proposal for revised clinical and electroencephalographic classification of epileptic seizures. Epilepsia 1981;22:489-501.

2. Commission on Classification and Terminology of the International League Against Epilepsy. Proposal for revised classification of epilepsies and epileptic syndromes. Epilepsia 1989;30:389-399.

3. Panayiotopoulos CP, Tahan R, Obeid T. Juvenile myoclonic epilepsy: factors of error involved in the diagnosis and treatment. Epilepsia 1991;32:672-676.

4. Panayiotopoulos CP, Obeid T, Waheed G. Differentiation of typical absence seizures in epileptic syndromes: a video EEG study of 224 seizures in 20 patients. Brain 1989;112:1039-1056.

5. Aliberti V, Grunewald RA, Panayiotopoulos CP, Chroni E. Focal electroencephalographic abnormalities in juvenile myoclonic epilepsy. Epilepsia 1994;35:297-301. 
6. Panayiotopoulos CP, Koutroumanidis M, Giannakodimos S, Agathonikou A. Idiopathic generalised epilepsy in adults manifested by phantom absences, generalised tonic-clonic seizures, and frequent absence status. J Neurol Neurosurg Psychiatry 1997;63:622-627.

7. Berkovic SF, Andermann F, Andermann E, Gloor P. Concepts of absence epilepsies: discrete syndromes or biological continuum. Neurology 1987;37:993-1000.

8. American Electroencephalographic Society. Guideline seven: a proposal for standard montages to be used in clinical EEG. J Clin Neurophysio 1994;11:30-36.

9. Panayiotopoulos CP. Perioral myoclonia with abscences: a new syndrome. In Wolf P. (ed) Epileptic seizures and syndromes. London: John Libbey \& Company, 1994:143-153.

10. Appleton RE, Panayiotopoulos CP, Acomb BA, Beirne M. Eyelid myoclonia with typical absences: an epilepsy syndrome. J Neurol Neurosurg Psychiatry 1993;56:1312-1316.

11. Williamson P, Spencer SS. Clinical and EEG features of complex partial seizures of extratemporal origin. Epilepsia 1986; 27(Suppl):46-63.

12. Quesney LF, Cendes F, Olivier A, Dubeau F, Andermann F. Intracranial electroencephalographic investigation in frontal lobe epilepsy. Adv Neurol 1995;66:243-258

13. Panayiotopoulos CP, Chroni E, Daskalopoulos C, Baker A, Rowlinson S, Walsh P. Typical absence seizures in adults: clinical, EEG, video-EEG findings and diagnostic/syndromic considerations. J Neurol Neurosurg Psychiatry 1992;55:1002-1008.

14. Gelisse P, Genton P, Thomas P, Rey M, Samuelian IC, Dravet C. Clinical factors of drug resistance in juvenile myoclonic epilepsy. J Neurol Neurosurg Psychiatry 2001;70:240-243.

15. Lombroso CT. Consistent EEG focalities detected in subjects with primary generalized epilepsies monitored for two decades. Epilepsia 1997;38:797-812.

16. Janz D. Juvenile myoclonic epilepsy: epilepsy with impulsive petit mal. Cleve Clin J Med 1989;56 (Suppl ):S23-33.
17. Yacubian EMT. Epilepsia mioclonica juvenil. In Guerreiro CAM, Guerreiro MM, Cendes F, Lopes-Cendes I (eds.). Epilepsia. São Paulo: Lemos Editorial, 2000:215-222.

18. Montalenti E, Imperiale D, Rovera A, Bergamasco B, Benna P. Clinical features, EEG findings and diagnostic pitfalls in juvenile myoclonic epilepsy: a series of 63 patients. J Neurol Sci 2001;184:65-70.

19. Janz D. The idiopathic generalized epilepsies of adolescence with childhood and juvenile age of onset. Epilepsia 1997;38:4-11.

20. Panayiotopoulos CP, Obeid T, Tahan AR. Juvenile myoclonic epilepsy: a 5-year prospective study. Epilepsia 1994;35:285-296.

21. Delgado-Escueta AV, Enrile-Bacsal F. Juvenile myoclonic epilepsy of Janz. Neurology 1984;34:285-294

22. Capovilla G, Rubboli G, Beccaria F, Lorenzetti ME, Montagnini A, Resi C et al. A clinical spectrum of the myoclonic manifestations associated with typical absences in childhood absence epilepsy. A video-polygraphic study. Epileptic Disord 2001;3:57-62

23. King MA, Newton MR, Jackson GD, et al. Epileptology of the first-seizure presentation: a clinical, electroencephalographic, and magnetic resonance imaging study of 300 consecutive patients. Lancet 1998; 352:1007-1011.

24. Meencke HJ, Janz D. Neuropathological findings in primary generalized epilepsy: a study of eight cases. Epilepsia 1984;25:8-21.

25. Opeskin K, Kalnins RM, Halliday G, Cartwright H, Berkovic SF. Idiopathic generalized epilepsy: lack of significant microdysgenesis. Neurology 2000;55:1101-1106.

26. Genton $P$, Gelisse $P$, Thomas $P$, Dravet $C$. Do carbamazepine and phenytoin aggravate juvenile myoclonic epilepsy? Neurology 2000;55:1106-1109.

27. Fernando-Dongas MC, Radtke RA, VanLandingham KE, Husain AM Characteristics of valproic acid resistant juvenile myoclonic epilepsy. Seizure 2000;9:385-388

28. Genton P. When antiepileptic drugs aggravate epilepsy. Brain Dev 2000;22:75-80.

29. Liporace JD, Sperling MR, Dichter MA. Absence seizures and carbamazepine in adults. Epilepsia 1994;35:1026-1028. 